\title{
Pacemaker lead-induced tricuspid stenosis treated with percutaneous valvotomy
}

\author{
Nilüfer Çetiner ${ }^{1 \oplus}$, Işıl Yıldırım Baştuhan ${ }^{1 \odot}$, Tolga Özyiğit' ${ }^{2 \odot}$, Ferit Onur Mutluer ${ }^{3 \odot}$, \\ Alpay Çeliker ${ }^{1 \odot}$
}

Departments of ${ }^{1}$ Pediatric Cardiology and ${ }^{3}$ Cardiology Koç University Faculty of Medicine, İstanbul; ${ }^{2}$ Department of Cardiology, Amerikan Hospital, İstanbul, Turkey.

\begin{abstract}
Background. Tricuspid stenosis is an uncommon complication of ventricular pacemaker electrode implantation, with few cases reported in the literature.

Case. We present an 18-year-old male who developed severe tricuspid stenosis 15 years after endocardial VVI pacemaker implantation for complete AV block following a surgically repaired ventricular septal defect.

Conclusion. In this case we have shown that percutaneous balloon valvuloplasty was performed and successful in treating this complication.
\end{abstract}

Key words: congenital heart defect, pacemaker, percutaneous balloon valvuloplasty, tricuspid stenosis.

Tricuspid stenosis is a rare late complication of endocardial pacemaker implantation that occurs generally in middle-aged patients. ${ }^{1}$ As there are limited reports of treatment and outcome of tricuspid stenosis in the literature, we present a young man with endocardial pacemaker-induced symptomatic tricuspid stenosis which was treated by percutaneous balloon valvuloplasty.

\section{Case Report}

An 18-year-old male presented to our clinic with exertional fatigue. His medical history was remarkable for VVI transvenous pacemaker implantation at 3 years of age because of a thirddegree atrioventricular block that occurred after a surgically repaired ventricular septal

\footnotetext{
Nilüfer Çetiner

nilufercetiner@hotmail.com
}

Received 25th December 2019, revised 27th March 2020, accepted 20th April 2020.

This case report was presented at the 15th National Congress of Pediatric Cardiology and Cardiac Surgery, Antalya, Turkey, 2016 (P-236). defect at 9 months of age. The patient received a diagnosis of tricuspid valve (TV) stenosis associated with a pacemaker implantation at 11 years of age, which worsened during followup. The transvenous pacemaker was replaced with an epicardial pacemaker to solve the transvenous lead related problem. The distal part of the electrode could not be removed due to firm adherence to the TV apparatus. At current presentation, he had no complaints of fever or weight loss. Physical examination revealed a loud middiastolic murmur and a 2/6 pansystolic murmur on the tricuspid region. He had no hepatomegaly, lower limb edema and jugular venous distension. The blood chemistry findings were unremarkable. Echocardiography revealed right atrial dilatation with dilated hepatic veins and vena cava inferior. On transthoracic echocardiography the thickened septal leaflet of the TV was noted (Fig. 1). Doppler interrogation revealed peak and mean gradients of 17,6 and $10 \mathrm{mmHg}$, respectively, across the TV and color flow imaging showed moderate tricuspid regurgitation (Figs. 2 and 3), along with mild mitral regurgitation. Echocardiographic findings were suggestive 


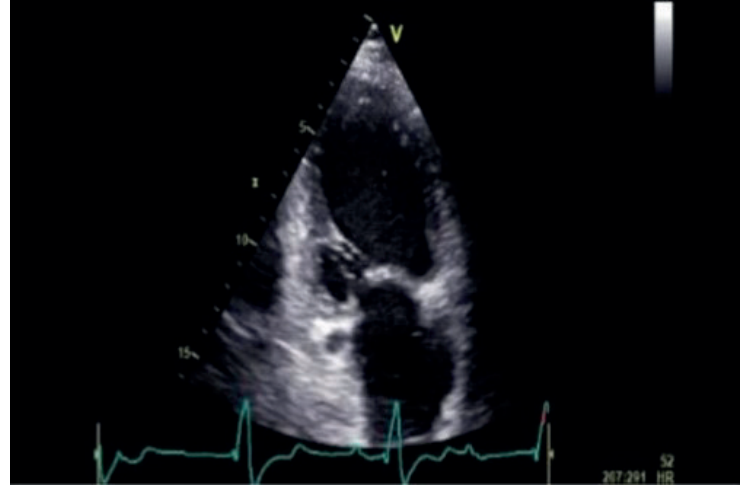

Fig. 1. Thickened septal leaflet of tricuspid valve.

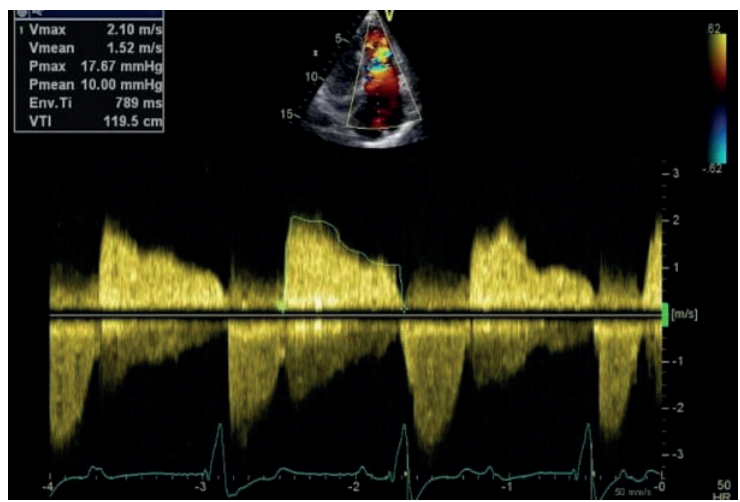

Fig. 2. Doppler Echocardiogram showing gradient across the tricuspid valve.

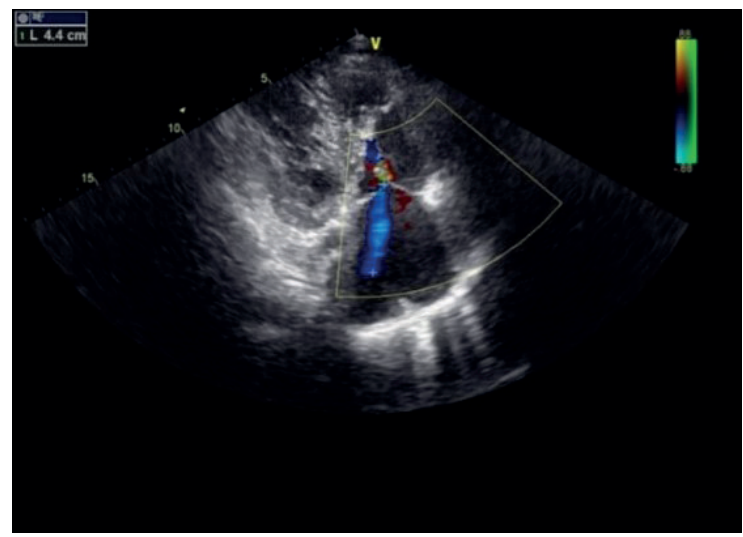

Fig. 3. Echocardiogram showing moderate tricuspid regurgitation.

of persistent and worsening TV stenosis. Percutaneous tricuspid valvuloplasty (PTV) was performed guided by $2 \mathrm{D}$ and $3 \mathrm{D}$ transesophageal echocardiography (TEE) under general anesthesia. TEE showed the stenotic annulus of TV (Fig. 4). The maximum and mean
TV gradients on TEE were 13 and $7 \mathrm{mmHg}$, respectively. The TV area using planimetry and annulus were measured as $0,9 \mathrm{~cm}^{2}$ and $34,5 \mathrm{~mm}$, respectively. Cardiac catheterization showed an increased right atrium pressure (mean 10 $\mathrm{mm} \mathrm{Hg}$ ) and normal pulmonary artery and right ventricular pressures. The valve was dilated after four attempts of inflation with a 28-mm Inoue balloon (Figs. 5 and 6). After final inflation, a transvalvular maximum gradient of $6 \mathrm{mmHg}$ and a mean gradient of $3 \mathrm{~mm} \mathrm{Hg}$ were measured by echocardiography. On 3D TEE an enlarged tricuspid annulus was noted (Fig. 7). The TV area was measured as $1,9 \mathrm{~cm}^{2}$ on 3D TEE. There was no increase in valve regurgitation. The patient was discharged after 24 hours without complications and remained asymptomatic, with a mean gradient across the TV of $3 \mathrm{mmHg}$ at two years of follow-up. Consent for this case report was received from the family.

\section{Discussion}

Tricuspid stenosis is an uncommon complication of ventricular pacemaker implantation, with few cases reported in the literature and generally occurs in middle-aged patients. The usual mechanisms described are right ventricular inflow obstruction by TV vegetations (i.e. infective endocarditis), or multiple pacemaker leads and fibrosis secondary to mechanical trauma. The resulting endothelial damage triggers a series of local events, including chronic inflammation, fibrosis, calcification and valve stenosis. ${ }^{1,2}$ The present patient was young and the most probable etiology of tricuspid stenosis was endothelial damage and fibrosis of the TV septal leaflet secondary to leaflet perforation by the lead.

Most patients are asymptomatic for a long time and present late with right heart failure. ${ }^{3}$ The present case had exertional fatigue with echocardiographic findings of significant TV stenosis.

In most previous literature, the therapeutic management of the tricuspid stenosis revolves 


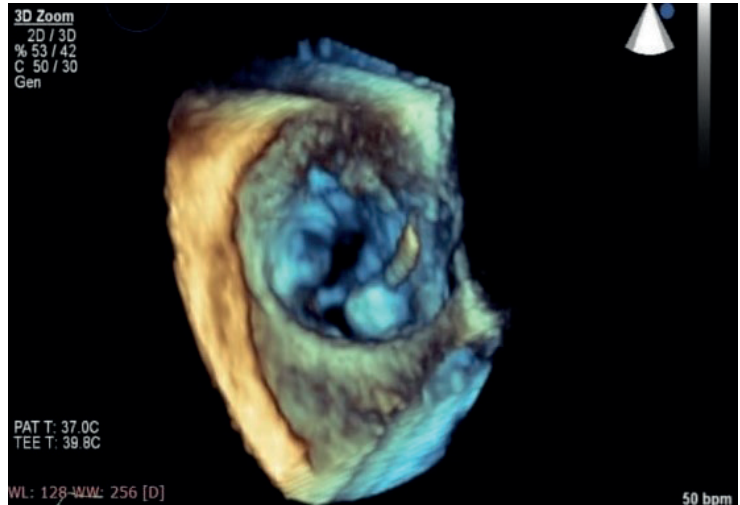

Fig. 4. TEE showing the stenotic annulus of tricuspid valve.

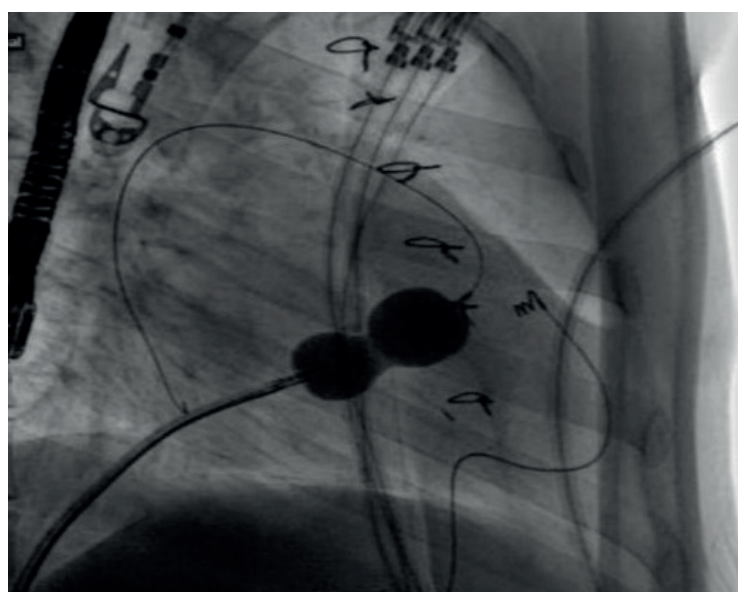

Fig. 5. Image of dilated balloon in the early period during valvuloplasty.

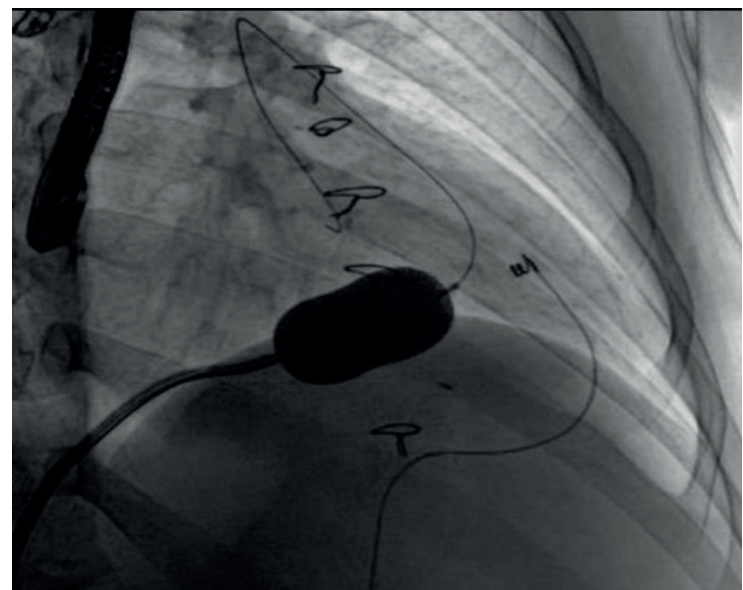

Fig. 6. Image of dilated balloon in the late period during valvuloplasty.

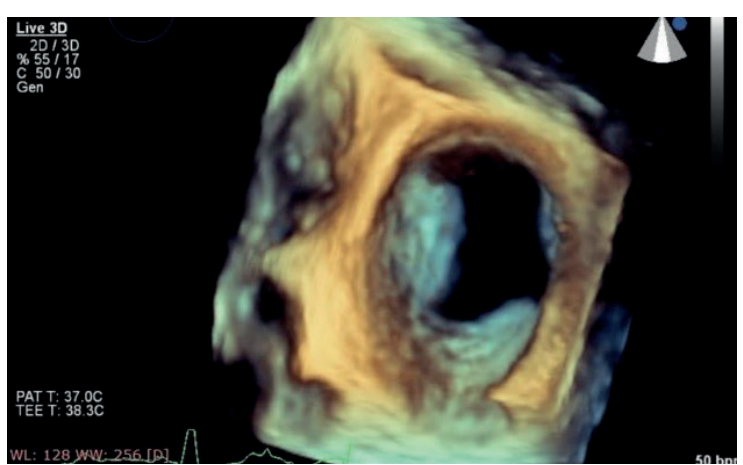

Fig. 7. 3D TEE showing the enlarged tricuspid annulus.

around decongestive therapy and surgical lead removal with TV replacement. ${ }^{3}$ The reported management of these cases has included medical management in three (offer of surgery declined in two), surgical lead removal and TV replacement in two, and surgical lead removal followed by tricuspid valvuloplasty in one. ${ }^{2}$

Percutaneous management has also been lately reported, and shown to be safe and effective. ${ }^{2,4-6}$ Limited data are available on longterm outcomes. A report of four patients with severe TS who were successfully treated with balloon valvotomy found that symptomatic and hemodynamic improvements persisted at six months follow-up., ${ }^{2,-6}$ In the present case the treatment was performed by successful balloon valvuloplasty. In view of the lasting reduction in gradient by balloon valvuloplasty, no further intervention has been necessary. After treatment the patient was asymptomatic and at two years of follow-up the mean gradient across the TV was $3 \mathrm{mmHg}$.

\section{REFERENCES}

1. Rosenberg Y, Myatt JP, Feldman M, et al. Down to the wire: tricuspid stenosis in the setting of multiple pacing leads. Pacing Clin Electrophysiol 2010; 33: e49-e52.

2. Hussain T, Knight WB, McLeod KA. Lead-induced tricuspid stenosis-successful management by balloon angioplasty. Pacing Clin Electrophysiol 2009; 32: 140-142. 
3. Krishnan A, Moulick A, Sinha P, et al. Severe tricuspid valve stenosis secondary to pacemaker leads presenting as ascites and liver dysfunction: a complex problem requiring a multidisciplinary therapeutic approach. J Interv Card Electrophysiol 2009; 24: 71-75.

4. Rama-Merchan JC, Arribas-Jimenez A, MartinMoreiras J, Garcia-Fernandez E, Cruz-Gonzalez I. Pacemaker lead-related tricuspid stenosis successfully treated with percutaneous balloon valvuloplasty guided by 3D echocardiography. Rev Port Cardiol 2014; 33: 739.e1-e3.
5. Michiels V, Delabays A, Eeckhout E. Percutaneous balloon valvotomy for the treatment of pacemaker lead-induced tricuspid stenosis. Heart 2014; 100: 352.

6. Patil DV, Nabar AA, Sabnis GR, Phadke MS, Lanjewar CP, Kerkar PG. Percutaneous tricuspid valvotomy for pacemaker lead-induced tricuspid stenosis. Indian Heart J 2015; 67(Suppl 3): S115-S116. 